Article

\title{
Certain Chebyshev-Type Inequalities Involving Fractional Conformable Integral Operators
}

\author{
Gauhar Rahman ${ }^{1}\left(\mathbb{D}\right.$, Zafar Ullah ${ }^{2}$, Aftab Khan ${ }^{3}$, Erhan Set ${ }^{4, *(1)}$ and \\ Kottakkaran Sooppy Nisar ${ }^{5}$ (i) \\ 1 Department of Mathematics, Shaheed Benazir Bhutto University, Sharingal, Upper Dir, \\ Khyber Pakhtoon Khwa 18000, Pakistan; gauhar55uom@gmail.com \\ 2 Department of Mathematics, University of Education Lahore, Dera Ghazi Khan Campus 54770, Pakistan; \\ zafarbhatti73@gmail.com \\ 3 Department of Mathematics, Shaheed Benazir Bhutto University, Sharingal, Upper Dir, \\ Khyber Pakhtoon Khwa 18000, Pakistan; aftabm84@gmail.com or aftab@sbbu.edu.pk \\ 4 Faculty of Science and Arts, Department of Mathematics, Ordu University, Ordu 52000, Turkey \\ 5 Department of Mathematics, College of Arts and Sciences, Prince Sattam Bin Abdulaziz University, \\ Wadi Aldawaser 11991, Saudi Arabia; ksnisar1@gmail.com or n.sooppy@psau.edu.sa \\ * Correspondence: erhanset@yahoo.com
}

Received: 2 April 2019; Accepted: 16 April 2019; Published: 21 April 2019

\begin{abstract}
Since an interesting functional by P.L. Chebyshev was presented in the year 1882, many results, which are called Chebyshev-type inequalities, have been established. Some of these inequalities were obtained by using fractional integral operators. Very recently, a new variant of the fractional conformable integral operator was introduced by Jarad et al. Motivated by this operator, we aim at establishing novel inequalities for a class of differentiable functions, which are associated with Chebyshev's functional, by employing a fractional conformable integral operator. We also aim at showing important connections of the results here with those including Riemann-Liouville fractional and classical integrals.
\end{abstract}

Keywords: Riemann-Liouville (R-L) fractional integral; fractional conformable integral; Chebyshev's functional; differentiable functions; integral inequalities

MSC: 26A33; 26D10

\section{Introduction}

In applied mathematics, fractional calculus (FC) is an influential branch that deals with derivatives and integrals of arbitrary orders [1]. Recently, many theories and results have been presented in this field, but still, there are huge amounts of non-local phenomena to be unexplored and waiting to be found. The FC has many applications in various fields of physical sciences, especially in diffusion equations [2,3]. The contemporary progress of FC in different fields is available in many recent papers [4-9].

In [10], the Chebyshev functional for two integrable functions $g$ and $h$, which are synchronous (i.e., $(g(x)-g(y))(h(x)-h(y)) \geq 0$, for any $x, y \in[a, b])$ on $[a, b]$, is defined as:

$$
T(g, h)=\frac{1}{b-a} \int_{a}^{b} g(\tau) h(\tau) d \tau-\frac{1}{b-a}\left(\int_{a}^{b} g(\tau) d \tau\right) \frac{1}{b-a}\left(\int_{a}^{b} h(\tau) d \tau\right) .
$$


The functional (1) has attracted many researcher's attention due to applications in mathematics and statistics and a number of inequalities associated with (1) (see, e.g., [11-15]). In [16] (also see [10]), a variant of the Chebyshev functional was defined by:

$$
T(g, h, f)=\int_{a}^{b} f(\tau) d \tau \int_{a}^{b} f(\tau) g(\tau) h(\tau) d \tau-\int_{a}^{b} f(\tau) g(\tau) d \tau \int_{a}^{b} f(\tau) h(\tau) d \tau
$$

where $g$ and $h$ are integrable on $[a, b]$ and $f$ is a positive and integrable function on $[a, b]$. The applications of the functional defined in (2) are found in probability and statistical problems. Its further applications in differential and integral equations are found in [17-19]. In [11,12,20-24], several inequalities related to the functional (2) were given. In [15], Dragomir obtained the resulting inequality linked to this functional:

$$
|T(g, h, f)| \leq\left\|g^{\prime}\right\|\left\|h^{\prime}\right\|\left[\int_{a}^{b} f(\tau) d \tau \int_{a}^{b} \tau^{2} f(\tau) d \tau-\left(\int_{a}^{b} \tau f(\tau) d \tau\right)^{2}\right]
$$

where $g$ and $h$ are differentiable and $g^{\prime}, h^{\prime} \in L_{\infty}(a, b)$, and $f$ is positive and integrable on $[a, b]$. Throughout this paper, we will use the space of all continuous functions $C([0, \infty))$ from $[0, \infty)$ into $\mathbb{R}$ and $L_{\infty}([0, \infty))$ the space of all bounded functions $g(\tau)$ on $[0, \infty)$, with the norm defined by:

$$
\|g\|_{\infty}=\sup _{\tau \in[0, \infty[}|g(\tau)|
$$

Recently, Jarad et al. [25] introduced new fractional integral operators, which are a generalization of R-L, Hadamard, and Katugampola fractional integrals through the idea of fractional integral operators given by Abdeljawad [26]. Dahmani [27] developed some Chebyshev-type inequalities in the case of two differentiable functions using the R-L fractional integrals. Here, motivated actually by the works of Dahmani [27] and Jarad et al. [25], we aim at establishing several Chebyshev-type inequalities associated with fractional conformable integrals by using differentiable functions. Some special cases of these results are also considered. For our design, we recall some definitions and properties as follows [28]:

Definition 1. A real-valued function $g(\tau), \tau \geq 0$ is known to be in the space $C_{\mu}, \mu \in \mathbb{R}$ if there exist $p \in \mathbb{R}$ such that $p>\mu$ and $g(\tau)=\tau^{p} g_{1}(\tau)$ where $g_{1}(\tau) \in C([0, \infty))$.

Definition 2. A function $g(\tau), \tau \geq 0$ is known to be in the space $C_{\mu}^{n}, \mu \in \mathbb{R}$, if $g^{(n)} \in C_{\mu}$.

Definition 3. The $R$-L fractional integral operator of order $\gamma \geq 0$, for a function $g \in C_{\mu},(\mu \geq-1)$, is given by:

$$
\mathfrak{I}^{\gamma} g(x)=\frac{1}{\Gamma(\gamma)} \int_{a}^{x}(x-t)^{\gamma-1} g(t) d t
$$

where the gamma function $\Gamma$ is given in [29].

In a similar manner, the definition of the fractional conformable integral can be given as:

Definition 4. The fractional conformable integral $\beta \mathfrak{I}^{\alpha}$ of order $\beta>0$, for a function $g \in C_{\mu},(\mu \geq-1)$, is defined by:

$$
\beta \mathfrak{I}^{\alpha} g(\tau)=\frac{1}{\Gamma(\beta)} \int_{0}^{\tau}\left(\frac{\tau^{\alpha}-t^{\alpha}}{\alpha}\right)^{\beta-1} t^{\alpha-1} g(t) d t ; \alpha>0
$$

Clearly, one can get ${ }^{0} \mathfrak{I}^{\alpha} g(\tau)=g(\tau)$ and: 


$$
\beta \mathfrak{I}^{\alpha} \gamma \mathfrak{I}^{\alpha} g(\tau)={ }^{\beta+\gamma} \mathfrak{I}^{\alpha} g(\tau)=\gamma \mathfrak{I}^{\alpha} \beta \mathfrak{I}^{\alpha} g(\tau) .
$$

For more details, the readers are referred to $[25,30]$.

Remark 1. The particular case of fractional conformable integral operator ${ }^{\beta} \mathfrak{I}^{\alpha} g(z)$ in (5) when $\alpha=1$ reduces directly to the Riemann-Liouville fractional operator.

\section{Main Results}

In this section, the Chebyshev-type integral inequalities for differentiable functions defined on $[0, \infty)$ involving the fractional conformable integral operator are introduced.

Theorem 1. Let $g$ and $h$ be two differentiable functions on $[0, \infty)$ such that $g^{\prime}, h^{\prime} \in L_{\infty}([0, \infty))$, then the following inequality holds for all $\tau>0, \alpha, \beta>0$ :

$$
\begin{aligned}
& \left|\frac{\tau^{\alpha \beta}}{\alpha^{\beta} \Gamma(\beta+1)} \beta \mathfrak{I}^{\alpha} g h(\tau)-\beta \mathfrak{I}^{\alpha} g(\tau)^{\beta} \mathfrak{I}^{\alpha} h(\tau)\right| \\
& \leq\left\|g^{\prime}\right\|_{\infty}\left\|h^{\prime}\right\|_{\infty}\left[\frac{\tau^{\alpha \beta}}{\alpha^{\beta} \Gamma(\beta+1)} \beta \mathfrak{I}^{\alpha} \tau^{2}-\left({ }^{\beta} \mathfrak{I}^{\alpha} \tau\right)^{2}\right] .
\end{aligned}
$$

Proof. Let:

$$
H(u, v)=(g(u)-g(v))(h(u)-h(v)) ; u, v \in(0, \tau) .
$$

Multiplying (7) by $\frac{1}{\Gamma(\beta)}\left(\frac{\tau^{\alpha}-u^{\alpha}}{\alpha}\right)^{\beta-1} u^{\alpha-1}$ and then integrating with respect to $u$ over $(0, \tau)$, we have:

$$
\begin{aligned}
& \frac{1}{\Gamma(\beta)} \int_{0}^{\tau}\left(\frac{\tau^{\alpha}-u^{\alpha}}{\alpha}\right)^{\beta-1} u^{\alpha-1} H(u, v) d u \\
= & { }^{\beta} \mathfrak{I}^{\alpha} g h(\tau)-g(v)^{\beta} \mathfrak{I}^{\alpha} h(\tau)-h(v)^{\beta} \mathfrak{I}^{\alpha} g(\tau)+g(v) h(v) \frac{\tau^{\alpha \beta}}{\alpha^{\beta} \Gamma(\beta+1)} .
\end{aligned}
$$

Again, multiplying (8) by $\frac{1}{\Gamma(\beta)}\left(\frac{\tau^{\alpha}-v^{\alpha}}{\alpha}\right)^{\beta-1} v^{\alpha-1}$ and then integrating with respect to $v$ over $(0, \tau)$, we have:

$$
\begin{aligned}
& \frac{1}{\Gamma^{2}(\beta)} \int_{0}^{\tau} \int_{0}^{\tau}\left(\frac{\tau^{\alpha}-u^{\alpha}}{\alpha}\right)^{\beta-1} u^{\alpha-1}\left(\frac{\tau^{\alpha}-v^{\alpha}}{\alpha}\right)^{\beta-1} v^{\alpha-1} H(u, v) d u d v \\
= & 2\left(\frac{\tau^{\alpha \beta}}{\alpha^{\beta} \Gamma(\beta+1)} \beta \mathfrak{J}^{\alpha} g h(\tau)-\beta \mathfrak{I}^{\alpha} g(\tau)^{\beta} \mathfrak{I}^{\alpha} h(\tau)\right) .
\end{aligned}
$$

Furthermore, on the other hand, we get:

$$
H(u, v)=\int_{u}^{v} \int_{u}^{v} g^{\prime}(x) h^{\prime}(\tau) d x d \tau .
$$

As $g^{\prime}, h^{\prime} \in L_{\infty}([0, \infty))$, therefore we have:

$$
|H(u, v)| \leq\left|\int_{u}^{v} g^{\prime}(x) d x\left\|\int_{u}^{v} h^{\prime}(\tau) d \tau \mid \leq\right\| g^{\prime}\left\|_{\infty}\right\| h^{\prime} \|_{\infty}(u-v)^{2} .\right.
$$


Thus, we can write:

$$
\begin{aligned}
& \frac{1}{\Gamma^{2}(\beta)} \int_{0}^{\tau} \int_{0}^{\tau}\left(\frac{\tau^{\alpha}-u^{\alpha}}{\alpha}\right)^{\beta-1} u^{\alpha-1}\left(\frac{\tau^{\alpha}-v^{\alpha}}{\alpha}\right)^{\beta-1} v^{\alpha-1}|H(u, v)| d u d v \\
\leq & \frac{\left\|g^{\prime}\right\|_{\infty}\left\|h^{\prime}\right\|_{\infty}}{\Gamma^{2}(\beta)} \int_{0}^{\tau} \int_{0}^{\tau}\left(\frac{\tau^{\alpha}-u^{\alpha}}{\alpha}\right)^{\beta-1} u^{\alpha-1}\left(\frac{\tau^{\alpha}-v^{\alpha}}{\alpha}\right)^{\beta-1} \\
& \times v^{\alpha-1}\left(u^{2}-2 u v+v^{2}\right) d u d v .
\end{aligned}
$$

From (12), we estimate the following inequality:

$$
\begin{aligned}
& \frac{1}{\Gamma^{2}(\beta)} \int_{0}^{\tau} \int_{0}^{\tau}\left(\frac{\tau^{\alpha}-u^{\alpha}}{\alpha}\right)^{\beta-1} u^{\alpha-1}\left(\frac{\tau^{\alpha}-v^{\alpha}}{\alpha}\right)^{\beta-1} v^{\alpha-1}|H(u, v)| d u d v \\
\leq & 2\left\|g^{\prime}\right\|_{\infty}\left\|h^{\prime}\right\|_{\infty}\left[\frac{\tau^{\alpha \beta}}{\alpha^{\beta} \Gamma(\beta+1)} \beta \mathfrak{I}^{\alpha} \tau^{2}-\left(\beta \mathfrak{I}^{\alpha} \tau\right)^{2}\right] .
\end{aligned}
$$

Hence, (9) and (13) give the desired results (6).

Theorem 2. Let $g$ and $h$ be two differentiable functions on $[0, \infty) \ni g^{\prime}, h^{\prime} \in L_{\infty}([0, \infty))$, then the subsequent inequality holds for all $\tau>0, \alpha, \beta, \mu>0$;

$$
\begin{aligned}
& \quad \frac{\tau^{\alpha \beta}}{\alpha^{\beta} \Gamma(\beta+1)} \beta^{\alpha} \mathfrak{I}^{\alpha} g h(\tau)+\frac{\tau^{\alpha \mu}}{\alpha^{\mu} \Gamma(\mu+1)}{ }^{\mu} \mathfrak{I}^{\alpha} g h(\tau) \\
& -{ }^{\beta} \mathfrak{I}^{\alpha} g(\tau)^{\mu} \mathfrak{I}^{\alpha} h(\tau)-{ }^{\mu} \mathfrak{I}^{\alpha} g(\tau)^{\beta} \mathfrak{I}^{\alpha} h(\tau) \mid \\
& \leq\left\|g^{\prime}\right\|_{\infty}\left\|h^{\prime}\right\|_{\infty}\left[\frac{\tau^{\alpha \beta}}{\alpha^{\beta} \Gamma(\beta+1)}{ }^{\mu} \mathfrak{I}^{\alpha} \tau^{2}-2\left({ }^{\beta} \mathfrak{I}^{\alpha} \tau\right)\left({ }^{\mu} \mathfrak{I}^{\alpha} \tau\right)+\frac{\tau^{\alpha \mu}}{\alpha^{\mu} \Gamma(\mu+1)} \beta \mathfrak{I}^{\alpha} \tau^{2}\right] .
\end{aligned}
$$

Proof. Multiplying (8) by $\frac{1}{\Gamma(\mu)}\left(\frac{\tau^{\alpha}-v^{\alpha}}{\alpha}\right)^{\mu-1} v^{\alpha-1}$ and then integrating with respect to $v$ over $(0, \tau)$, we have:

$$
\begin{aligned}
& \frac{1}{\Gamma(\beta) \Gamma(\mu)} \int_{0}^{\tau} \int_{0}^{\tau}\left(\frac{\tau^{\alpha}-u^{\alpha}}{\alpha}\right)^{\beta-1} u^{\alpha-1}\left(\frac{\tau^{\alpha}-v^{\alpha}}{\alpha}\right)^{\mu-1} v^{\alpha-1} H(u, v) d u d v \\
= & \left(\frac{\tau^{\alpha \beta}}{\alpha^{\beta} \Gamma(\beta+1)}{ }^{\mu} \mathfrak{I}^{\alpha} g h(\tau)+\frac{\tau^{\alpha \mu}}{\alpha^{\mu} \Gamma(\mu+1)} \beta \mathfrak{I}^{\alpha} g h(\tau)\right. \\
& \left.-{ }^{\beta} \mathfrak{I}^{\alpha} g(\tau)^{\mu} \mathfrak{I}^{\alpha} h(\tau)-{ }^{\mu} \mathfrak{I}^{\alpha} g(\tau)^{\beta} \mathfrak{I}^{\alpha} h(\tau)\right) .
\end{aligned}
$$

From Relation (11), we have:

$$
\begin{aligned}
& \frac{1}{\Gamma(\beta) \Gamma(\mu)} \int_{0}^{\tau} \int_{0}^{\tau}\left(\frac{\tau^{\alpha}-u^{\alpha}}{\alpha}\right)^{\beta-1} u^{\alpha-1}\left(\frac{\tau^{\alpha}-v^{\alpha}}{\alpha}\right)^{\mu-1} v^{\alpha-1}|H(u, v)| d u d v \\
& \leq \frac{\left\|g^{\prime}\right\|_{\infty}\left\|h^{\prime}\right\|_{\infty}}{\Gamma(\beta) \Gamma(\mu)} \\
& \times \int_{0}^{\tau} \int_{0}^{\tau}\left(\frac{\tau^{\alpha}-u^{\alpha}}{\alpha}\right)^{\beta-1} u^{\alpha-1}\left(\frac{\tau^{\alpha}-v^{\alpha}}{\alpha}\right)^{\mu-1} v^{\alpha-1}\left(u^{2}-2 u v+v^{2}\right) d u d v .
\end{aligned}
$$

From (15) and (16), we get the required result (14).

Remark 2. If we choose $\beta=\mu$ in Theorem 2, then we get Theorem 1.

Theorem 3. Let $g$ and $h$ be two differentiable functions on $[0, \infty)$ with $h^{\prime}(\tau) \neq 0, \tau \in[0, \infty)$. Let there exist $K>0$ such that $\frac{g^{\prime}(\tau)}{h^{\prime}(\tau)} \leq K$, then the subsequent inequality holds for all $\tau>0, \alpha, \beta, \mu>0$; 


$$
\begin{aligned}
& \mid \frac{\tau^{\alpha \beta}}{\alpha^{\beta} \Gamma(\beta+1)}{ }^{\beta} \mathfrak{I}^{\alpha} g h(\tau)+\frac{\tau^{\alpha \mu}}{\alpha^{\mu} \Gamma(\mu+1)}{ }^{\mu} \mathfrak{I}^{\alpha} g h(\tau) \\
& -{ }^{\beta} \mathfrak{I}^{\alpha} g(\tau)^{\mu} \mathfrak{I}^{\alpha} h(\tau)-{ }^{\mu} \mathfrak{I}^{\alpha} g(\tau)^{\beta} \mathfrak{I}^{\alpha} h(\tau) \mid \\
\leq & K\left[\frac{\tau^{\alpha \beta}}{\alpha^{\beta} \Gamma(\beta+1)}{ }^{\mu} \mathfrak{I}^{\alpha} h^{2}(\tau)-2\left({ }^{\beta} \mathfrak{I}^{\alpha} h(\tau)\right)\left({ }^{\mu} \mathfrak{I}^{\alpha} h(\tau)\right)+\frac{\tau^{\alpha \mu}}{\alpha^{\mu} \Gamma(\mu+1)} \beta \mathfrak{I}^{\alpha} h^{2}(\tau)\right] .
\end{aligned}
$$

Proof. Let $g$ and $h$ obey the conditions of Theorem 3. Then, from the generalization of Lagrange's mean value theorem, for every $u, v \in[0, \tau] ; h(u) \neq h(v), \tau>0$, there exist a constant $c$ between $u$ and $v$ such that:

$$
\frac{g(u)-g(v)}{h(u)-h(v)}=\frac{g^{\prime}(c)}{h^{\prime}(c)}
$$

Thus, for every $u, v \in[0, \tau]$, we have:

$$
|g(u)-g(v)| \leq K|h(u)-h(v)| .
$$

It follows that:

$$
|H(u, v)| \leq K(h(u)-h(v))^{2}
$$

Hence, we have:

$$
\begin{aligned}
& \frac{1}{\Gamma(\beta) \Gamma(\mu)} \int_{0}^{\tau} \int_{0}^{\tau}\left(\frac{\tau^{\alpha}-u^{\alpha}}{\alpha}\right)^{\beta-1} u^{\alpha-1}\left(\frac{\tau^{\alpha}-v^{\alpha}}{\alpha}\right)^{\mu-1} v^{\alpha-1}|H(u, v)| d u d v \\
& \leq \frac{K}{\Gamma(\beta) \Gamma(\mu)} \int_{0}^{\tau} \int_{0}^{\tau}\left(\frac{\tau^{\alpha}-u^{\alpha}}{\alpha}\right)^{\beta-1} u^{\alpha-1}\left(\frac{\tau^{\alpha}-v^{\alpha}}{\alpha}\right)^{\mu-1} \\
& \times v^{\alpha-1}\left(h^{2}(u)-2 h(u) h(v)+h^{2}(v)\right) d u d v .
\end{aligned}
$$

Hence, from (18), we get the required inequality.

Corollary 1. Let $g$ and $h$ be two differentiable functions on $[0, \infty)$ with $h^{\prime}(\tau) \neq 0, \tau \in[0, \infty)$. Let there exist $K>0$ such that $\frac{g^{\prime}(\tau)}{h^{\prime}(\tau)} \leq K$, then the following inequality holds for all $\tau>0, \alpha, \beta>0$ :

$$
\begin{aligned}
& \left|\frac{\tau^{\alpha \beta}}{\alpha^{\beta} \Gamma(\beta+1)} \beta \mathfrak{I}^{\alpha} g h(\tau)-\beta \mathfrak{I}^{\alpha} g(\tau)^{\beta} \mathfrak{I}^{\alpha} h(\tau)\right| \\
\leq K & \left.K \frac{\tau^{\alpha \beta}}{\alpha^{\beta} \Gamma(\beta+1)} \beta \mathfrak{I}^{\alpha} h^{2}(\tau)-\left(\beta \mathfrak{I}^{\alpha} h(\tau)\right)^{2}\right] .
\end{aligned}
$$

Proof. Taking $\mu=\beta$ in Theorem 3, we get the desired corollary.

Remark 3. If we consider $\alpha=1$ in Theorems 1-3, then we get the results of Dahmani [27]. Similarly, if we take $\alpha=\beta=1$, then we get the classical inequalities [15].

Now, we prove further generalization of Theorems 1 and 2.

Theorem 4. Let $f$ be a positive function on $[0, \infty)$ and $g$ and $h$ be two differentiable functions having the same sense of variation on $[0, \infty)$. If $g^{\prime}, h^{\prime} \in L_{\infty}([0, \infty))$, then the following inequality holds for all $\tau>0, \alpha, \beta>0$ :

$$
\begin{aligned}
& \quad 0 \leq{ }^{\beta} \mathfrak{I}^{\alpha} f(\tau)^{\beta} \mathfrak{I}^{\alpha} f g h(\tau)-{ }^{\beta} \mathfrak{I}^{\alpha} f g(\tau)^{\beta} \mathfrak{I}^{\alpha} f h(\tau) \\
& \leq\left\|g^{\prime}\right\|_{\infty}\left\|h^{\prime}\right\|_{\infty}\left[{ }^{\beta} \mathfrak{I}^{\alpha} f(\tau)^{\beta} \mathfrak{I}^{\alpha} \tau^{2} f(\tau)-\left({ }^{\beta} \mathfrak{I}^{\alpha} \tau f(\tau)\right)^{2}\right] .
\end{aligned}
$$


Proof. Define:

$$
\begin{gathered}
H(u, v)=(g(u)-g(v))(h(u)-h(v)) ; u, v \in(0, \tau), \tau>0 \\
=g(u) h(u)-g(u) h(v)-g(v) h(u)+g(v) h(u) .
\end{gathered}
$$

Since $g$ and $h$ satisfy the conditions of Theorem 4, therefore we have:

$$
H(u, v) \geq 0
$$

Multiplying both sides of (21) by $\frac{1}{\Gamma(\beta)} \int_{0}^{\tau}\left(\frac{\tau^{\alpha}-u^{\alpha}}{\alpha}\right)^{\beta-1} u^{\alpha-1} f(u)$ and integrating the resultant identity with respect to $u$ from $0-\tau$, we have:

$$
\begin{aligned}
& \frac{1}{\Gamma(\beta)} \int_{0}^{\tau}\left(\frac{\tau^{\alpha}-u^{\alpha}}{\alpha}\right)^{\beta-1} u^{\alpha-1} f(u) H(u, v) d u \\
= & { }^{\beta} \mathfrak{I}^{\alpha} f g h(\tau)-h(v)^{\beta} \mathfrak{I}^{\alpha} f g(\tau)-g(v)^{\beta} \mathfrak{I}^{\alpha} f h(\tau)+g(v) h(v)^{\beta} \mathfrak{I}^{\alpha} f(\tau) \geq 0 .
\end{aligned}
$$

Again, multiplying (23) by $\frac{1}{\Gamma(\beta)} \int_{0}^{\tau}\left(\frac{\tau^{\alpha}-v^{\alpha}}{\alpha}\right)^{\beta-1} v^{\alpha-1} f(v)$ and integrating the resultant identity with respect to $v$ from $0-\tau$, we have:

$$
\begin{aligned}
& \frac{1}{2 \Gamma^{2}(\beta)} \int_{0}^{\tau} \int_{0}^{\tau}\left(\frac{\tau^{\alpha}-u^{\alpha}}{\alpha}\right)^{\beta-1} u^{\alpha-1}\left(\frac{\tau^{\alpha}-v^{\alpha}}{\alpha}\right)^{\beta-1} v^{\alpha-1} f(u) h(v) H(u, v) d u d v \\
= & \beta \mathfrak{I}^{\alpha} f(\tau)^{\beta} \mathfrak{I}^{\alpha} f g h(\tau)-\beta \mathfrak{I}^{\alpha} f g(\tau)^{\beta} \mathfrak{I}^{\alpha} f h(\tau) \geq 0 .
\end{aligned}
$$

From (11), we have:

$$
\begin{aligned}
& \frac{1}{\Gamma^{2}(\beta)} \int_{0}^{\tau} \int_{0}^{\tau}\left(\frac{\tau^{\alpha}-u^{\alpha}}{\alpha}\right)^{\beta-1} u^{\alpha-1}\left(\frac{\tau^{\alpha}-v^{\alpha}}{\alpha}\right)^{\beta-1} v^{\alpha-1} f(u) f(v)|H(u, v)| d u d v \\
\leq & \frac{\left\|g^{\prime}\right\|_{\infty}\left\|h^{\prime}\right\|_{\infty}}{\Gamma^{2}(\beta)} \int_{0}^{\tau} \int_{0}^{\tau}\left(\frac{\tau^{\alpha}-u^{\alpha}}{\alpha}\right)^{\beta-1} u^{\alpha-1}\left(\frac{\tau^{\alpha}-v^{\alpha}}{\alpha}\right)^{\beta-1} v^{\alpha-1} f(u) f(v)\left(u^{2}-2 u v+v^{2}\right) d u d v .
\end{aligned}
$$

Consequently, it follows:

$$
\begin{aligned}
& \frac{1}{\Gamma^{2}(\beta)} \int_{0}^{\tau} \int_{0}^{\tau}\left(\frac{\tau^{\alpha}-u^{\alpha}}{\alpha}\right)^{\beta-1} u^{\alpha-1}\left(\frac{\tau^{\alpha}-v^{\alpha}}{\alpha}\right)^{\beta-1} v^{\alpha-1} f(u) f(v)|H(u, v)| d u d v \\
\leq & 2\left\|g^{\prime}\right\|_{\infty}\left\|h^{\prime}\right\|_{\infty}\left[\beta \mathfrak{I}^{\alpha} f(\tau)^{\beta} \mathfrak{I}^{\alpha} \tau^{2} f(\tau)-\left({ }^{\beta} \mathfrak{I}^{\alpha} \tau f(\tau)\right)^{2}\right] .
\end{aligned}
$$

According to (24) and (26), we get the desired proof.

Remark 4. Taking $H(\tau)=1$ in Theorem 4, we get Theorem 1. Similarly, taking $\alpha=\beta=1$, we get Inequality (2).

Theorem 5. Let the positive function on $[0, \infty)$ be $f$ and $g, h$ be two differentiable functions with the same sense of variation on $[0, \infty)$. If $g^{\prime}, h^{\prime} \in L_{\infty}([0, \infty))$, then the following inequality holds for all $\tau>0, \alpha, \beta, \mu>0$ :

$$
\begin{aligned}
& 0 \leq{ }^{\beta} \mathfrak{I}^{\alpha} f(\tau){ }^{\mu} \mathfrak{I}^{\alpha} f g h(\tau)+{ }^{\mu} \mathfrak{I}^{\alpha} f(\tau){ }^{\beta} \mathfrak{I}^{\alpha} f g h(\tau)-{ }^{\beta} \mathfrak{I}^{\alpha} f g(\tau){ }^{\mu} \mathfrak{I}^{\alpha} f h(\tau)-{ }^{\mu} \mathfrak{I}^{\alpha} f g(\tau)^{\beta} \mathfrak{I}^{\alpha} f h(\tau) \\
\leq & \left\|g^{\prime}\right\|_{\infty}\left\|h^{\prime}\right\|_{\infty}\left[{ }^{\beta} \mathfrak{I}^{\alpha} f(\tau)^{\mu} \mathfrak{I}^{\alpha} \tau^{2} f(\tau)-2\left({ }^{\beta} \mathfrak{I}^{\alpha} \tau f(\tau)\right)\left({ }^{\mu} \mathfrak{I}^{\alpha} \tau f(\tau)\right)+{ }^{\mu} \mathfrak{I}^{\alpha} f(\tau)^{\beta} \mathfrak{I}^{\alpha} \tau^{2} f(\tau)\right] .
\end{aligned}
$$

Proof. Define:

$$
H(u, v)=(g(u)-g(v))(h(u)-h(v)) ; u, v \in(0, \tau), \tau>0 .
$$


Multiplying $\frac{1}{\Gamma(\beta)} \int_{0}^{\tau}\left(\frac{\tau^{\alpha}-u^{\alpha}}{\alpha}\right)^{\beta-1} u^{\alpha-1} f(u)$ on both sides of (28) and integrating the resultant identity with respect to $u$ from $0-\tau$, we have:

$$
\begin{aligned}
& \frac{1}{\Gamma(\beta)} \int_{0}^{\tau}\left(\frac{\tau^{\alpha}-u^{\alpha}}{\alpha}\right)^{\beta-1} u^{\alpha-1} f(u) H(u, v) d u \\
= & { }^{\beta} \mathfrak{I}^{\alpha} f g h(\tau)-g(v)^{\beta} \mathfrak{I}^{\alpha} f g(\tau)-g(v)^{\beta} \mathfrak{I}^{\alpha} f h(\tau)+g(v) h(v)^{\beta} \mathfrak{I}^{\alpha} f(\tau) \geq 0 .
\end{aligned}
$$

Again, multiplying (29) by $\frac{1}{\Gamma(\mu)} \int_{0}^{\tau}\left(\frac{\tau^{\alpha}-v^{\alpha}}{\alpha}\right)^{\mu-1} v^{\alpha-1} f(v)$ and integrating the resultant identity with respect to $v$ from $0-\tau$, we have:

$$
\begin{aligned}
& \frac{1}{\Gamma(\beta) \Gamma(\mu)} \int_{0}^{\tau} \int_{0}^{\tau}\left(\frac{\tau^{\alpha}-u^{\alpha}}{\alpha}\right)^{\beta-1} u^{\alpha-1}\left(\frac{\tau^{\alpha}-v^{\alpha}}{\alpha}\right)^{\mu-1} v^{\alpha-1} f(u) f(v) H(u, v) d u d v \\
= & { }^{\mu} \mathfrak{I}^{\alpha} f(\tau)^{\beta} \mathfrak{I}^{\alpha} f g h(\tau)+{ }^{\beta} \mathfrak{I}^{\alpha} f(\tau)^{\mu} \mathfrak{I}^{\alpha} f g h(\tau)-{ }^{\beta} \mathfrak{I}^{\alpha} f g(\tau)^{\mu} \mathfrak{I}^{\alpha} f h(\tau)-{ }^{\mu} \mathfrak{I}^{\alpha} f g(\tau)^{\beta} \mathfrak{I}^{\alpha} f h(\tau) \geq 0 .
\end{aligned}
$$

From Relations (10) and (11), we have:

$$
\begin{aligned}
& \frac{1}{\Gamma(\beta) \Gamma(\mu)} \int_{0}^{\tau} \int_{0}^{\tau}\left(\frac{\tau^{\alpha}-u^{\alpha}}{\alpha}\right)^{\beta-1} u^{\alpha-1}\left(\frac{\tau^{\alpha}-v^{\alpha}}{\alpha}\right)^{\mu-1} v^{\alpha-1} f(u) f(v)|H(u, v)| d u d v \\
\leq & \frac{\left\|h^{\prime}\right\|_{\infty}\left\|h^{\prime}\right\|_{\infty}}{\Gamma(\beta) \Gamma(\mu)} \int_{0}^{\tau} \int_{0}^{\tau}\left(\frac{\tau^{\alpha}-u^{\alpha}}{\alpha}\right)^{\beta-1} u^{\alpha-1}\left(\frac{\tau^{\alpha}-v^{\alpha}}{\alpha}\right)^{\mu-1} v^{\alpha-1} f(u) f(v)\left(u^{2}-2 u v+v^{2}\right) d u d v \\
= & \left\|g^{\prime}\right\|_{\infty}\left\|h^{\prime}\right\|_{\infty}\left[{ }^{\beta} \mathfrak{I}^{\alpha} f(\tau)^{\mu} \mathfrak{I}^{\alpha} \tau^{2} f(\tau)+{ }^{\mu} \mathfrak{I}^{\alpha} f(\tau)^{\beta} \mathfrak{I}^{\alpha} \tau^{2} f(\tau)-2\left({ }^{\beta} \mathfrak{I}^{\alpha} \tau f(\tau)\right)\left({ }^{\mu} \mathfrak{I}^{\alpha} \tau f(\tau)\right)\right] .
\end{aligned}
$$

Hence, from (30) and (31), we get the desired proof.

Remark 5. Taking $\mu=\beta$, we get Theorem 4. Similarly, taking $f(\tau)=1$ in Theorem 5, we get Theorem 2 .

\section{Conclusions}

Inequalities for a class of differentiable functions that are connected to Chebyshev's functional by employing the fractional conformable integral operator are introduced in this paper. The inequalities achieved in this paper are more general than the existing classical inequalities cited herein. This work will reduce to the inequalities for a class of differentiable functions, which include the Riemann-Liouville fractional integral operator, by taking $\alpha=1$, which were presented earlier by [16,27]. Furthermore, one can get the classical results by taking $\alpha=\beta=1$, which were presented in [15].

Author Contributions: conceptualization, G.R. and K.S.N.; methodology, A.K.; validation, E.S. and Z.U.; formal analysis, K.S.N. and G.R. ; writing-original draft preparation, G.R. and K.S. N; writing-review and editing, K.S.N. and E.S.; supervision, A.K.; funding acquisition, Z.U.

Funding: This research received no external funding.

Acknowledgments: The First and third authors thank to Higher Education Commission of Pakistan for supporting this work under Start-up Research Grant Project.

Conflicts of Interest: The authors declare no conflict of interest.

\section{References}

1. Miller, K.S.; Ross, B. An Introduction to the Fractional Calculus and Fractional Differential Equations; Wiley-Interscience: Bellingham, DC, USA, 1993

2. Hristov, J. Approximate solutions to fractional subdiffusion equations. Eur. Phys. J. Spec. Top. 2011, 193, 229-243. [CrossRef] 
3. dos Santos, M.A. Fractional Prabhakar Derivative in Diffusion Equation with Non-Static Stochastic Resetting. Physics 2019, 1, 40-58. [CrossRef]

4. Fernandez, A.; Baleanu, D.; Srivastava, H.M. Series representations for fractional-calculus operators involving generalised Mittag-Leffler functions. Commun. Nonlinear Sci. Numer. Simul. 2019 67, 517-527 [CrossRef]

5. Hilfer, R.; Luchko, Y. Desiderata for Fractional Derivatives and Integrals. Mathematics 2019, 7, 149. [CrossRef]

6. Almeida, R.; Tavares, D.; Torres, D.F. The Variable-Order Fractional Calculus Of Variations; SpringerBriefs in Applied Sciences and Technology, Springer International Publishing: Cham Switzerland, 2018.

7. Araci, S.; Rahman, G.; Ghaffar, A.; Nisar, K.S. Fractional Calculus of Extended Mittag-Leffler Function and Its Applications to Statistical Distribution. Mathematics 2019, 7, 248. [CrossRef]

8. Huang, C.-J.; Rahman, G.; Nisar, K.S.; Ghaffar, A.; Qi, F. Some Inequalities of the Hermite-Hadamard Type for k-Fractional Conformable Integrals. Aust. J. Math. Anal. Appl. 2019, 16, 1-9.

9. Sontakke, B.; Shaikh, A.; Nisar, K.S. Existence and uniqueness of integrable solutions of fractional order initial value equations. J. Math. Model. 2018, 6, 137-148.

10. Chebyshev, P.L. Sur les expressions approximatives des integrales definies par les autres prises entre les mêmes limites. Proc. Math. Soc. Charkov 1882, 2, 93-98

11. Anastassiou, G.; Hooshmandasl, M.R.; Ghasemi, A.; Moftakharzadeh, F. Montgomery identities for fractional integrals and related fractional inequalities. J. Inequal. Pure Appl. Math. 2009, 10, 1-16.

12. Belarbi, S.; Dahmani, Z. On some new fractional integral inequalities. J. Inequal. Pure Appl. Math. 2009, 10, 1-5.

13. Baleanu, D.; Purohit, S.D.; Agarwal, P. On fractional integral inequalities involving hypergeometric operators. Chin. J. Math. 2014, 2014, 609476. [CrossRef]

14. Dahmani, Z.; Tabharit, L.; Taf, S. New inequalities via Riemann-Liouville fractional integration. J. Adv. Res. Sci. Computing 2010, 2, 40-45.

15. Dragomir, S.S. Some integral inequalities of Gruss type. Indian J. Pure Appl. Math. 2002, 31, 397-415.

16. Dahmani, Z. The Riemann-Liouville Operator to Generate Some New Inequalities. Int. J. Nonlinear Sci. 2011, 12, 452-455.

17. Cerone, P.; Dragomir, S.S. A refinement of the Gruss inequality and applications. Tamkang J. Math. 2007, 38, $37-49$.

18. Dragomir, S.S. A generalization of Gruss's inequality in inner product spaces and applications. J. Math. Anal. Appl. 1999, 237, 74-82. [CrossRef]

19. Mitrinovic, D.S.; Pecaric, J.E.; Fink, A.M. Classical and new inequalities in Analysis; Kluwer Academic Publishers: Dordrecht, The Netherlands, 1993.

20. Mercer, M.A. An improvement of the Gruss inequality. J. Inequal. Pure Appl. Math. 2005, 6, 1-4.

21. Mercer, M.A.; Mercer, P. New proofs of the Gruss inequality. Aust. J. Math. Anal. Appl. 2004, 1, 12.

22. Niculescu, C.P.; Persson, L.E. Convex Functions And Their Applications; A Contemporary Approach, CMS Books in Mathematics; Springer Verlag: New York, NY, USA, 2006; Volume 23,

23. Pachpatte, B.G. On multidimensional Gruss type integral inequalities. J. Inequal. Pure Appl. Math. 2002, 3, $1-15$.

24. Sarikaya, M.Z.; Aktan, N.; Yildirim, H. On weighted Chebyshev-Gruss like inequalities on time scales. J. Math. Ineq. 2008, 2, 185-195. [CrossRef]

25. Jarad, F.; Uğurlu, E.; Abdeljawad, T.; Baleanu, D. On a new class of fractional operators. Adv. Differ. Equ. 2017, 2017, 1-16. [CrossRef]

26. Abdeljawad, T. On conformable fractional calculus. J. Comput. Appl. Math. 2015, 279, 57-66. [CrossRef]

27. Dahmani, Z. New inequalities for a class of differentiable functions. Int. J. Nonlinear Anal. Appl. 2011, 2, 19-23.

28. Gorenflo, R.; Mainardi, F. Fractional Calculus: Integral and Differential Equations of Fractional Order; Springer Verlag: Wien, Austria, 1997; pp. 223-276.

29. Srivastava, H.M.; Choi, J. Zeta and q-Zeta Functions and Associated Series and Integrals; Elsevier Science Publishers: Amsterdam, The Netherlands; London, UK; New York, NY, USA, 2012. 
30. Set, E.; Mumcu, İ.; Özdemir, M.E. Grüss type inequalities involving new conformable fractional integral operators. AIP Conf. Proc. 2018, 1991, 020020; doi:10.1063/1.5047893. [CrossRef]

(C) 2019 by the authors. Licensee MDPI, Basel, Switzerland. This article is an open access article distributed under the terms and conditions of the Creative Commons Attribution (CC BY) license (http://creativecommons.org/licenses/by/4.0/). 